\title{
YANG-MILLS DETOUR COMPLEXES AND CONFORMAL GEOMETRY
}

\author{
A. ROD GOVER, PETR SOMBERG AND VLADIMÍR SOUČEK
}

\begin{abstract}
Working over a pseudo-Riemannian manifold, for each vector bundle with connection we construct a sequence of three differential operators which is a complex (termed a Yang-Mills detour complex) if and only if the connection satisfies the full Yang-Mills equations. A special case is a complex controlling the deformation theory of Yang-Mills connections. In the case of Riemannian signature the complex is elliptic. If the connection respects a metric on the bundle then the complex is formally self-adjoint. In dimension 4 the complex is conformally invariant and generalises, to the full Yang-Mills setting, the composition of (two operator) Yang-Mills complexes for (anti-)self-dual Yang-Mills connections. Via a prolonged system and tractor connection a diagram of differential operators is constructed which, when commutative, generates differential complexes of natural operators from the Yang-Mills detour complex. In dimension 4 this construction is conformally invariant and is used to yield two new sequences of conformal operators which are complexes if and only if the Bach tensor vanishes everywhere. In Riemannian signature these complexes are elliptic. In one case the first operator is the twistor operator and in the other sequence it is the operator for Einstein scales. The sequences are detour sequences associated to certain Bernstein-Gelfand-Gelfand sequences.
\end{abstract}

\section{IntRODUCTION}

In the study of Riemannian and pseudo-Riemannian geometry it is often valuable to use differential operators with good conformal behaviour. In the Riemannian setting, elliptic differential operators are particularly important. For example the conformal Laplacian controls the conformal variation of the scalar curvature. This was exploited heavily in the solution by Schoen, Aubin, Trudinger, and Yamabe (see [40]) of the "Yamabe Problem" of finding, via conformal rescaling, constant scalar curvature metrics on compact manifolds. Related curvature prescription problems and techniques have exploited the higher order conformal Laplacians of Paneitz, Graham et al. [35, 8, 18]. These operators on functions (or really densities) also find a natural place in the recent developments $[24,36]$ concerning the asymptotics and scattering theory of the conformally compact Poincaré-Einstein metric of Fefferman-Graham [23].

On many tensor and spinor fields there is no conformally invariant elliptic operator (taking values in an irreducible bundle); this follows from the classification

ARG would like to thank the Royal Society of New Zealand for support via Marsden Grants no. 02-UOA-108 and 06-UOA-029. Part of the work was prepared during a visit of ARG supported by the E. Čech Center. PS and VS acknowledge the support of the grant GA CR 201/05/2117 and the grant MSM 021620839. 
of conformally invariant differential operators on the sphere [7, 22]. This classification is based on the structure of generalised Verma modules and from this it follows that often the analogue, or replacement, for a conformal elliptic operator on the sphere is an elliptic complex of conformally invariant differential operators. However the situation is complicated for conformally curved structures. The requirement that a sequence of differential operators be both conformally invariant and form a complex is severe. On the other hand when such complexes exist they can be expected to play a serious role in treating the underlying structure. This idea is already well-established in the setting of self-dual 4-manifolds $[1,19]$. On fully conformally curved $n$-manifolds, with $n$ even, there is a class of elliptic conformal complexes on differential forms [11]. Each of these is different to the de Rham complex, and these complexes generalise the conformally invariant operator of [35], with leading term $\Delta^{n / 2}$. Another class of complexes is based around the (Fefferman-Graham) obstruction tensor [23]. This is a natural conformal 2-tensor that generalises, to higher even dimensions, the Bach tensor in dimension 4. It turns out that the formal deformations of obstruction-flat manifolds are controlled by a sequence of conformal operators, which form an elliptic complex if and only if the structure is obstruction-flat [12]. Unfortunately there is no obvious way to generalise either the construction in [11], or that in [12].

For 4-manifolds we construct here two conformal differential sequences which are (formally self-adjoint) complexes if and only if the (conformally invariant) Bach-tensor [2] vanishes everywhere. This condition is weaker than self-duality. In fact conformally Einstein manifolds are also Bach-flat and there are structures which are Bach-flat and neither conformally-Einstein nor half-flat [30]. Writing $\mathbf{T}: \mathbb{S} \rightarrow \mathrm{Tw}$ for the usual twistor operator on Dirac spinors (as in e.g. [5]), in Theorem 4.5 we obtain a differential complex

$$
\mathbb{S} \stackrel{\mathbf{T}}{\rightarrow} \mathrm{Tw} \stackrel{M^{\Sigma}}{\rightarrow} \mathrm{Tw} \stackrel{\mathbf{T}^{*}}{\rightarrow} \mathbb{S}
$$

where $M^{\Sigma}$ is a third order Rarita-Schwinger type operator. On the other hand in Theorem 4.3 we construct

$$
\mathcal{E}^{0} \stackrel{P}{\rightarrow} \mathcal{E}^{1,1} \stackrel{M^{\mathbb{T}}}{\rightarrow} \mathcal{E}^{1,1} \stackrel{P^{*}}{\rightarrow} \mathcal{E}^{0}
$$

where $M^{\mathbb{T}}$ is a second order conformal operator, similar in form to the operator which controls deformations of Einstein structures (see [6] and references therein), while $P$ is a curvature modification of the trace-free covariant Hessian. Nonvanishing solutions of $P$ give conformal factors $\sigma$ so that $\sigma^{-2} g$ is Einstein (see $[3])$; we show via the second sequence that the Bach tensor obstructs solutions. If the manifold is Riemannian then both of the complexes are elliptic. We have been intentionally explicit in treating these constructions, as it seems these complexes should play a fundamental role in conformal and Riemannian geometry. In the compact and Riemannian-signature setting the ellipticity implies that the complexes have finite dimensional cohomology spaces. In both cases the interpretation of the $0^{\text {th }}$-cohomology is well-known but as far as we know the first cohomology 
is a new global conformal invariant of Bach-flat structures. Such conformal elliptic complexes have the scope to yield further geometric information through their detour torsion invariants [10].

In fact the Theorems 4.3 and 4.5 construct short detour complexes in all dimensions $n \geq 3$ and $n \geq 4$ respectively. These complexes are conformally invariant only in dimension 4 , but by construction have a simple conformal behaviour and may well be of interest for physics in the Lorentzian setting.

The route to the constructions and results mentioned above is really one of the main points of the article. We believe that it lays foundations for an eventual general treatment of a large class of related complexes, and also many of the results should be of independent interest. The simplest example of a detour complex is the Maxwell detour complex

$$
\mathcal{E}^{0} \stackrel{d}{\rightarrow} \mathcal{E}^{1} \stackrel{\delta d}{\rightarrow} \mathcal{E}^{1} \stackrel{\delta}{\rightarrow} \mathcal{E}^{0}
$$

For each vector bundle $V$ and connection $D$ we construct, in section 3.1, a curvature adjusted twisting of this complex with the property that it is again a complex if and only if the connection $D$ is a (pure) Yang-Mills connection, see Theorem 3.2. In dimension 4 the resulting complexes are conformal. In sections 3.2 (in particular Theorem 3.4) we recover a class of these complexes by considering deformations of Yang-Mills connections. We show in Proposition 3.6 of Section 3.4 that, in dimension 4, the Yang-Mills detour complex generalises the composition of subcomplexes of Yang-Mills complexes arising from (anti-)self-dual connections. The next main item is a rather general construction, see diagram [D] in Section 4. This enables the Yang-Mills detour complex to be "translated" to yield new complexes. Broadly the motivational idea is this. If one has an overdetermined differential operator (of finite type) $\mathcal{B}^{0} \rightarrow \mathcal{B}^{1}$ then one may sometimes obtain a corresponding invariant connection on a prolonged system [33]. If the latter satisfies the Yang-Mills equations and, say, preserves a metric on the prolonged system then the Yang-Mills detour complex on the prolonged system descends and extends $\mathcal{B}^{0} \rightarrow \mathcal{B}^{1}$ to a complex. In reality this is an over-simplification, but it contains the germ of the main idea.

The first author would like to thank Helga Baum, Kengo Hirachi, Paul-Andi Nagy and Andrew Waldron for illuminating discussions.

\section{BACKGROUND: CONFORMAL GEOMETRY}

Recall that a conformal structure of signature $(p, q)$ on $M$ is a smooth ray subbundle $\mathcal{Q} \subset S^{2} T^{*} M$ whose fibre over $x$ consists of conformally related signature$(p, q)$ metrics at the point $x$ (and $S^{2} T^{*} M$ is the symmetric part of $\otimes^{2} T^{*} M$ ). Sections of $\mathcal{Q}$ are metrics $g$ on $M$. So we may equivalently view the conformal structure as the equivalence class $[g]$ of these conformally related metrics. The principal bundle $\pi: \mathcal{Q} \rightarrow M$ has structure group $\mathbb{R}_{+}$, and so each representation $\mathbb{R}_{+} \ni x \mapsto x^{-w / 2} \in \operatorname{End}(\mathbb{R})$ induces a natural line bundle on $(M,[g])$ that we term the conformal density bundle $E[w]$. We shall write $\mathcal{E}[w]$ for the space of sections of this bundle and $\boldsymbol{g}$ denotes the conformal metric, that is the tautological section of $S^{2} T^{*} M \otimes E[2]$ determined by the conformal structure. On conformal manifolds 
this will be used to identify $T M$ with $T^{*} M[2]$. Note $E[w]$ is trivialised by a choice of metric $g$ from the conformal class, and we write $\nabla$ for the connection corresponding to this trivialisation (and term this the Levi-Civita connection on $E[w]$ ). It follows that (the coupled) $\nabla_{a}$ preserves the conformal metric.

In dimensions $n \geq 3$ the Riemannian curvature can be decomposed into the totally trace-free Weyl curvature $C_{a b c d}$ and a remaining part described by the symmetric Schouten tensor $\mathrm{P}_{a b}$, according to $R_{a b c d}=C_{a b c d}+2 \boldsymbol{g}_{c[a} \mathrm{P}_{b] d}+2 \boldsymbol{g}_{d[b} \mathrm{P}_{a] c}$, where $[\cdots]$ indicates antisymmetrisation over the enclosed indices. The Schouten tensor is a trace modification of the Ricci tensor $\mathrm{Ric}_{a b}$ and vice versa: $\mathrm{Ric}_{a b}=$ $(n-2) \mathrm{P}_{a b}+\boldsymbol{J} \boldsymbol{g}_{a b}$, where we write $\mathrm{J}$ for the trace $\mathrm{P}_{a}{ }^{a}$ of $\mathrm{P}$. The Cotton tensor and Bach tensor are defined by, respectively,

$$
A_{a b c}:=2 \nabla_{[b} \mathrm{P}_{c] a} \quad \text { and } \quad B_{a b}:=\nabla^{c} A_{a c b}+\mathrm{P}^{d c} C_{d a c b} .
$$

Under a conformal transformation we replace a choice of metric $g$ by the metric $\hat{g}=e^{2 \omega} g$, where $\omega$ is a smooth function. Explicit formulae for the corresponding transformation of the Levi-Civita connection and its curvatures are given in e.g. $[3,32]$. We recall that, in particular, the Weyl curvature is conformally invariant $\widehat{C}_{a b c d}=C_{a b c d}$. In dimension $4 B_{a b}$ is conformally invariant.

We will write $\mathcal{E}^{k}[w]$ for the sections of the tensor product $E^{k}[w]:=\wedge^{k} T^{*} M \otimes$ $E[w]$. On conformal manifolds we use the notation $\mathcal{E}_{k}$ to mean the space of sections of $E_{k}:=\wedge^{k} T^{*} M \otimes E[2 k-n]$. This notation (following [11]) is suggested by the duality between the section spaces $\mathcal{E}^{k}$ and $\mathcal{E}_{k}$; compactly supported sections pair globally by contraction and integration. For any vector bundle $V, \mathcal{E}^{k}(V)$ is the space of smooth sections of $E^{k}(V):=\wedge^{k} T^{*} M \otimes V$, while $\mathcal{E}_{k}(V)$ means the space of sections of $E_{k}(V):=\wedge^{k} T^{*} M \otimes E[2 k-n] \otimes V$. When a metric from the conformal class is fixed, these spaces will be identified.

In conformal geometry the de Rham complex is a prototype for a class of sequences of bundles and conformally invariant differential operators, each of the form

$$
\mathcal{B}^{0} \rightarrow \mathcal{B}^{1} \rightarrow \cdots \rightarrow \mathcal{B}^{n}
$$

where the vector bundles $\mathcal{B}^{i}$ are irreducible tensor-spinor bundles. On the $n$-sphere there is one such complex for each irreducible module $\mathbb{V}$ for the group $G=S O(n+$ 1,1 ) of conformal motions, the space of solutions of the first (overdetermined) conformal operator $\mathcal{B}^{0} \rightarrow \mathcal{B}^{1}$ is isomorphic to $\mathbb{V}$, and the sequence gives a resolution of this space viewed as a sheaf. These are the conformal cases of the (generalised) Bernstein-Gelfand-Gelfand (BGG) sequences, a class of sequences of differential operators that exist on any parabolic geometry [7, 15]. As well as the operators $D_{i}: \mathcal{B}^{i} \rightarrow \mathcal{B}^{i+1}$ of the BGG sequence, in even dimensions there are conformally invariant "long operators" $L_{k}: \mathcal{B}^{k} \rightarrow \mathcal{B}^{n-k}$ for $k=1, \cdots, n / 2-1$ [7]. Thus there are sequences of the form

$$
\mathcal{B}^{0} \stackrel{D_{\rho}}{\rightarrow} \mathcal{B}^{1} \stackrel{D_{1}}{\rightarrow} \ldots \stackrel{D_{k-1}}{\rightarrow} \mathcal{B}^{k} \stackrel{L_{k}}{\rightarrow} \mathcal{B}^{n-k} \stackrel{D_{n-k}}{\rightarrow} \ldots \stackrel{D_{n-1}}{\rightarrow} \mathcal{B}^{n} .
$$

and, following $[11,10]$, we term these detour sequences since, in comparison to the BGG sequence, the long operator here bypasses the middle of the BGG sequence. Once again from the classification it follows that these detour sequences are in 
fact complexes in the case that the structure is conformally flat. The dimension 4 conformal complexes, constructed in Theorems 4.3 and 4.5 below, are detour sequences of this form with $k=1$.

\section{YANG-Mills Detour COMPleXes}

3.1. The general construction. We work over a pseudo-Riemannian $n$-manifold $(M, g)$ of signature $(p, q)(n \geq 2)$. Let $V$ denote a vector bundle with a connection $D$. We denote by $F$ the curvature of $D$. We also write $D$ for the induced connection on the dual bundle $V^{*}$. We write $d^{D}$ for the connection-coupled exterior derivative operator $d^{D}: \mathcal{E}^{k}(V) \rightarrow \mathcal{E}^{k+1}(V)$. Of course we could equally consider $d^{D}: \mathcal{E}^{k}\left(V^{*}\right) \rightarrow \mathcal{E}^{k+1}\left(V^{*}\right)$, and for the formal adjoint of this we write $\delta^{D}: \mathcal{E}_{k+1}(V) \rightarrow \mathcal{E}_{k}(V)$.

Let us write $F$. for the action of the curvature on the twisted 1-forms, $F$. : $\mathcal{E}^{1}(V) \rightarrow \mathcal{E}_{1}(V)$ given by $(F \cdot \varphi)_{a}:=F_{a}^{b} \varphi_{b}$ where we have indicated the abstract form indices explicitly, whereas the standard $\operatorname{End}(V)$ action of the curvature on the $V$-valued 1 -form is implicit. Using this we construct a differential operator

$$
M^{D}: \mathcal{E}^{1}(V) \rightarrow \mathcal{E}_{1}(V)
$$

by $M^{D} \varphi=\delta^{D} d^{D} \varphi-F \cdot \varphi$.

The operator $M^{D}$ has the property that its composition with $d^{D}$ is given simply by an algebraic action of the "Yang-Mills current" $\delta^{D} F$ on the bundle $V$, as follows.

Lemma 3.1. The composition of $M^{D}: \mathcal{E}^{1}(V) \rightarrow \mathcal{E}_{1}(V)$ with $d^{D}: \mathcal{E}^{0}(V) \rightarrow \mathcal{E}^{1}(V)$ is given by the exterior action of $\delta^{D} F$, as an $\operatorname{End}(V)$-valued 1-form:

$$
M^{D} d^{D}=\varepsilon\left(\delta^{D} F\right) .
$$

The composition of $\delta^{D}: \mathcal{E}_{1}(V) \rightarrow \mathcal{E}_{0}(V)$ with $M^{D}: \mathcal{E}^{1}(V) \rightarrow \mathcal{E}_{1}(V)$ is given by the interior action of $-\delta^{D} F$, as an $E^{1}$-valued endomorphism of $E^{1}(V)$ :

$$
\delta^{D} M^{D}=-\iota\left(\delta^{D} F\right)
$$

In these expressions the interior multiplication (indicated by $\iota(\cdot))$ and the exterior multiplication (indicated $\varepsilon(\cdot))$ refers to the form index of $\delta^{D} F$.

Proof: For the connection $D$ coupled with the Levi-Civita connection $\nabla$, let us also write $D$. Then, again using the notation where we exhibit abstract tensor indices but suppress indices for the bundle $V$, a formula for $M^{D}$ on a twisted 1 -form $\Psi_{a}$ is

$$
\left(M^{D} \Psi\right)_{b}=-D^{a} D_{a} \Psi_{b}+D^{a} D_{b} \Psi_{a}-F_{b}{ }^{a} \Psi_{a},
$$

since the Levi-Civita connection is torsion-free. On the other hand for $\Phi \in \mathcal{E}^{0}(V)$, $\left(d^{D} \Phi\right)_{a}=D_{a} \Phi$. Thus

$$
\begin{aligned}
\left(M^{D} d^{D} \Phi\right)_{b} & =D^{a}\left(D_{b} D_{a} \Phi-D_{a} D_{b} \Phi\right)-F_{b}{ }^{a} D_{a} \Phi \\
& =D^{a} F_{b a} \Phi-F_{b a} D^{a} \Phi \\
& =\left(\varepsilon\left(\delta^{D} F\right) \Phi\right)_{b} .
\end{aligned}
$$


By a similar calculation (or using the above on $\left(V^{*}, D\right)$ and taking formal adjoints) we obtain,

$$
\delta^{D} M^{D} \Psi=-\iota\left(\delta^{D} F\right) \Psi,
$$

for $\Psi \in \mathcal{E}_{1}(V)$. (Note that $\iota\left(\delta^{D} F\right) \Psi=-\left(D^{b} F_{b}{ }^{a}\right) \Psi_{a}$.)

Remark: Note that to simplify the punctuation in calculations, we often view sections of vector bundles as order 0 operators. Thus for example $D^{a} F_{b a} \Phi$ has the same meaning as $D^{a}\left(F_{b a} \Phi\right)$.

If the connection $D$ is orthogonal or unitary for some inner product or Hermitian form on $V$ (then $V$ may be identified with $V^{*}$ and) the algebraic action $F$ : $\mathcal{E}^{1}(V) \rightarrow \mathcal{E}_{1}(V)$ is easily verified to be formally self-adjoint and so, in this case, $M^{D}$ is formally self-adjoint. From these observations, and the Lemma 3.1, we have the following.

Theorem 3.2. The sequence of operators,

$$
\mathcal{E}^{0}(V) \stackrel{d^{D}}{\rightarrow} \mathcal{E}^{1}(V) \stackrel{M^{D}}{\rightarrow} \mathcal{E}_{1}(V) \stackrel{\delta^{D}}{\rightarrow} \mathcal{E}_{0}(V)
$$

is a complex if and only if the curvature $F$ of the connection $D$ satisfies the (pure) Yang-Mills equation

$$
\delta^{D} F=0
$$

In addition:

(i) If $D$ is an orthogonal or unitary connection then the sequence is formally selfadjoint.

(ii) In Riemannian signature the sequence is elliptic.

(iii) In dimension 4 the sequence (3) is conformally invariant.

Proof: It remains to show (ii) and (iii). For $(i i)$ we need that the symbol sequence is exact. This sequence is simply a tensor product twisting by $V$ of the symbol sequence of the Maxwell detour complex (1) and so it is sufficient to check that case. But that case is an easy consequence of the algebraic Hodge decomposition on an inner product space.

The conformally well-defined formal adjoint of the exterior derivative $d: \mathcal{E}^{k} \rightarrow$ $\mathcal{E}^{k+1}$ acts

$$
\delta: \mathcal{E}_{k+1} \rightarrow \mathcal{E}_{k}
$$

(cf. e.g. [11].) Note that in even dimensions on middle order forms we have $\mathcal{E}^{n / 2}=$ $\mathcal{E}_{n / 2}$ and so $\delta: \mathcal{E}^{n / 2} \rightarrow \mathcal{E}_{n / 2-1}$ is conformally invariant. The invariance persists if we twist by a connection $D$, and so from the definition of $M^{D}$ we have the result.

For a given connection $D$ on a vector bundle $V$, such that $\delta^{D} F=0$, we will term the complex (3) of Theorem 3.2 the (corresponding) Yang-Mills detour complex.

If $D$ is a Yang-Mills connection on a vector bundle $V$, then the dual connection on $V^{*}$ and the tensor product connection on any tensor power of these are also Yang-Mills. One might alternatively work with principal connections. If $\omega$ is a Yang-Mills connection on a principal bundle $\mathcal{P}$ with structure group $G$, then we obtain a complex (3) for every finite dimensional representation of $G$. 
3.2. A variational construction of the deformation detour. Returning to the general situation that began section 3.1, let $V$ denote a vector bundle with a connection $D$ and denote by $F$ the curvature of $D$. Consider now a smoothly parametrised family of connections $D^{t}$ (on $V$ ) given, on a section $v \in \mathcal{E}^{0}(V)$, by

$$
D_{a}^{t} v=D_{a} v+A_{a}^{t} v
$$

where for each $t \in \mathbb{R}, A^{t} \in \mathcal{E}^{1}(\operatorname{End} V)$ and $A^{0}=0$. With $F^{t}$ denoting the curvature of $D^{t}$, we have

$$
F_{a b}^{t}=F_{a b}+D_{a} A_{b}^{t}-D_{b} A_{a}^{t}+\left[A_{a}^{t}, A_{b}^{t}\right]
$$

where, once again, we write $D$ also to mean the connection on $V$ coupled with the Levi-Civita connection. It follows that the derivative of $F^{t}$ at $D=D^{0}$ is

$$
\dot{F}_{a b}=D_{a} \dot{A}_{b}-D_{b} \dot{A}_{a} \quad \text { where } \quad \dot{A}_{a}:=\left.\frac{d}{d t} A_{a}^{t}\right|_{t=0}
$$

that is $\dot{F}=d^{D} \dot{A}$. Now we calculate the derivative, at $D$, of $\delta^{D^{t}} F^{t}$. We have

$$
\begin{aligned}
\left.\frac{d}{d t} g^{a b} D_{a}^{t} F_{b c}^{t}\right|_{t=0} & =D^{b} \dot{F}_{b c}+\left[\dot{A}^{b}, F_{b c}\right] \\
& =D^{b}\left(D_{b} \dot{A}_{c}-D_{c} \dot{A}_{b}\right)+\left[F_{c}^{b}, \dot{A}_{b}\right]
\end{aligned}
$$

where $\dot{A}$ acts on $F$ and vice versa by the obvious composition of bundle endomorphisms. Note that, since the 1 -form $\dot{A}$ has values in End $V$, the last term here is $F \cdot \dot{A}$. Multiplying the display by -1 gives

$$
\left.\frac{d}{d t} \delta^{D^{t}} F^{t}\right|_{t=0}=M^{D} \dot{A}
$$

So we have, in particular, the following outcome.

Lemma 3.3. If $D$ is a Yang-Mills connection then the infinitesimal deformation $\dot{A}$ of $D$ is through Yang-Mills connections if and only if $M^{D} \dot{A}=0$.

In the vector bundle picture, a so-called gauge transformation arises locally by acting on $V$ by a section $u$ of the fibre bundle $\operatorname{Aut}(V)$ of invertible elements in $\operatorname{End}(V)$. From the Leibniz rule for $D$ (viewed as a connection on the tensor powers of $V$ and $V^{*}$ ) it follows immediately that this pulls back to a transformation

$$
D_{a} \mapsto D_{a}+u^{-1} D_{a} u,
$$

of the connection, and whence

$$
F_{a b} \mapsto u^{-1} F_{a b} u, \quad \text { and } \quad D^{a} F_{a b} \mapsto u^{-1}\left(D^{a} F_{a b}\right) u
$$

Thus if $u_{s}$ is a smoothly parametrised family of such transformations with $u_{0}=i d_{V}$ and derivative

$$
\left.\frac{d}{d s} u_{s}\right|_{s=0}=\dot{u} \in \mathcal{E}^{0}(\operatorname{End}(V))
$$

then we obtain that the infinitesimal variation of $D^{s}$ is exactly $d^{D} \dot{u}$ :

$$
\dot{D}_{a}=D_{a} \dot{u}
$$


So from this and (5) we have

$$
\left.\frac{d}{d s} \delta^{D^{s}} F^{s}\right|_{s=0}=M^{D} d^{D} \dot{u}
$$

On the other hand from (6) and (7) we get

$$
\left.\frac{d}{d s} \delta^{D^{s}} F^{s}\right|_{s=0}=\left(\delta^{D} F\right) \dot{u}-\dot{u} \delta^{D} F .
$$

Putting the last two results together brings us to

$$
M^{D} d^{D} \dot{u}=\varepsilon\left(\delta^{D} F^{\operatorname{End}(V)}\right) \dot{u}
$$

where $F^{\operatorname{End}(V)}$ is the curvature of $D$ viewed as a connection on $\operatorname{End}(V)$ (so e.g. $\left.F^{\operatorname{End}(V)} \dot{u}=[F, \dot{u}]\right)$. This agrees precisely with the specialisation of Lemma 3.1 to $\operatorname{End}(V)$ equipped with the connection induced from $D$ on $V$. In particular if $D$ is a Yang-Mills connection then so is the connection on $\operatorname{End}(V)$. Since $\operatorname{End}(V)$ carries the non-degenerate symmetric pairing $(U, W)=\operatorname{Tr}(U W)$ and this is preserved by $D$, then it follows from (7) that $M^{D}$ is formally self-adjoint with respect to the global pairing obtained by integrating $($,$) . (The point is that the Yang-Mills$ equations are the Euler-Lagrange equations for the Lagrangian density $\operatorname{Tr}\left(F^{a b} F_{a b}\right)$. So (7) shows that $M^{D}$ is the second variation of an action. By interchanging orders of variation one obtains the symmetry.) Thus from $M^{D} d^{D}$ we also have $\delta^{D} M^{D}=0$ and the following result.

Theorem 3.4. For a vector bundle V, with Yang-Mills connection D, the (formal) deformation detour complex

$$
\mathcal{E}^{0}(\operatorname{End}(V)) \stackrel{d^{D}}{\rightarrow} \mathcal{E}^{1}(\operatorname{End}(V)) \stackrel{M^{D}}{\rightarrow} \mathcal{E}_{1}(\operatorname{End}(V)) \stackrel{\delta^{D}}{\rightarrow} \mathcal{E}_{0}(\operatorname{End}(V))
$$

is formally self-adjoint. Its first cohomology $H^{1}(\operatorname{End}(V), D)$ is the formal tangent space at $D$ to the moduli space of Yang-Mills connections on $V$.

It follows from a general deformation theory that the complex (8) controls the full formal deformation theory of the Yang-Mills equations.

\subsection{Examples: (pseudo-)Riemannian manifolds with harmonic curva-} ture. On a pseudo-Riemannian (spin) manifold we write $\nabla$ for the Levi-Civita connection and $R$ for its curvature, the Riemannian curvature tensor. Riemannian structures satisfying $\delta^{\nabla} R=0$ are said to have harmonic curvature. Einstein manifolds, for example, are harmonic in this sense. There is a rich theory of harmonic manifolds, see [6] and references therein.

If $\delta^{\nabla} R=0$ then, from Theorem 3.2, we get a detour complex (3) for $V$ any tensor (spin) bundle. For example if $T M$ is the tangent bundle then we have

$$
M^{\nabla}: \mathcal{E}^{1}(T M) \rightarrow \mathcal{E}_{1}(T M) \quad \text { by } \quad S_{b}{ }^{c} \mapsto-2 \nabla^{a} \nabla_{[a} S_{b]}{ }^{c}-R_{b a}{ }^{c} S^{a d} .
$$

This annihilates the covariant derivative of any tangent vector field. 
3.4. Half-flat connections. In the setting of conformal (or pseudo-Riemannian) 4-manifolds, we observe here that when a vector bundle connection $D$ is half-flat then there is very simple interpretation of the Yang-Mills detour complex. First we review, in our current notation, some relevant (well-known) background.

Recall that on a conformal 4-manifold $\mathcal{M}$ of signature $(p, q)$ we have $\star \star=$ $(-1)^{k(4-k)+q}$ on $k$-forms. In the case of Minkowskian signature let us write $E_{ \pm}^{2}$ for the $\pm i$-eigenspaces of $\star$. In the other signatures $E_{ \pm}^{2}$ means the \pm 1 eigenspaces of $\star$. In any case, since $\star$ is a symmetric endomorphism of $E^{2}$, the decomposition of $E^{2}$ into $E_{+}^{2} \oplus E_{-}^{2}$ is orthogonal. Viewing the curvature $F$ (of $D$ on $V$ ) as a twisted 2-form, recall that the curvature, or the connection, is said to be self-dual (respectively anti-self-dual) if the component of $F$ in $\mathcal{E}_{-}^{2}(\operatorname{End}(V)$ ) (respectively in $\left.\mathcal{E}_{+}^{2}(\operatorname{End}(V))\right)$ is zero, $F_{-}=0$ (respectively $F_{+}=0$ ). So if a connection $D$ is half-flat, in this sense, then $\delta^{D} F$ is a multiple of $\star d^{D} F$. But this vanishes by the differential Bianchi identity for $F$. So $\delta^{D} F=0$ for connections which are either self-dual or anti-self-dual and each case gives a special setting where the sequence (3) is a complex.

Let us write $d_{ \pm}^{D}$ for the compositions given by $d^{D}: \mathcal{E}^{1}(U) \rightarrow \mathcal{E}^{2}(U)$ followed by the projections $\mathcal{E}^{2}(U) \rightarrow \mathcal{E}_{ \pm}^{2}(U)$, where $U$ means either the bundle $V$ or its dual $V^{*}$. Thus by construction the operators $d_{ \pm}^{D}: \mathcal{E}^{1}(U) \rightarrow \mathcal{E}_{ \pm}^{2}(U)$ are conformally invariant. We write $\delta_{ \pm}^{D}: \mathcal{E}_{ \pm}^{2}(V) \rightarrow \mathcal{E}_{1}(V)$ for the operators formally adjoint to $d_{ \pm}^{D}: \mathcal{E}^{1}\left(V^{*}\right) \rightarrow \mathcal{E}_{ \pm}^{2}\left(V^{*}\right)$. By construction these also are conformally invariant. Now on $\Phi \in \mathcal{E}^{0}(V)$ we have $d^{D} d^{D} \Phi=F \Phi$. The projection of this into $\mathcal{E}_{ \pm}^{2}$ vanishes for all $\Phi$ if and only if $F_{ \pm}=0$. By a similar observation for the composition $d^{D} d^{D}$ on $\mathcal{E}^{0}\left(V^{*}\right)$, and then taking formal adjoints, we see that we have the situation in the following proposition. These results are well-known.

Proposition 3.5. The sequences

$$
\mathcal{E}^{0}(V) \stackrel{d^{D}}{\longrightarrow} \mathcal{E}^{1}(V) \stackrel{d_{+}^{D}}{\longrightarrow} \mathcal{E}_{+}^{2}(V) \text { and } \mathcal{E}_{+}^{2}(V) \stackrel{\delta_{+}^{D}}{\longrightarrow} \mathcal{E}_{1}(V) \stackrel{\delta^{D}}{\longrightarrow} \mathcal{E}_{0}(V)
$$

are complexes if and only if $F_{+}=0$. Similarly the sequences

$$
\mathcal{E}^{0}(V) \stackrel{d^{D}}{\longrightarrow} \mathcal{E}^{1}(V) \stackrel{d_{-}^{D}}{\longrightarrow} \mathcal{E}_{-}^{2}(V) \text { and } \mathcal{E}_{-}^{2}(V) \stackrel{\delta_{-}^{D}}{\longrightarrow} \mathcal{E}_{1}(V) \stackrel{\delta^{D}}{\longrightarrow} \mathcal{E}_{0}(V)
$$

are complexes if and only if $F_{-}=0$. In Riemannian signature each of these is an elliptic complex.

Evidently then we obtain detour complexes by composing the twisted de Rham subcomplexes in the Proposition. For example if the connection $D$ is anti-self-dual then there is a detour complex

$$
\mathcal{E}^{0}(V) \stackrel{d^{D}}{\longrightarrow} \mathcal{E}^{1}(V) \stackrel{2 \delta_{+}^{D} d_{+}^{D}}{\longrightarrow} \mathcal{E}_{1}(V) \stackrel{\delta^{D}}{\longrightarrow} \mathcal{E}_{0}(V) .
$$

Similarly if $D$ is instead self-dual then there is a detour complex

$$
\mathcal{E}^{0}(V) \stackrel{d^{D}}{\longrightarrow} \mathcal{E}^{1}(V) \stackrel{2 \delta_{-}^{D} d_{-}^{D}}{\longrightarrow} \mathcal{E}_{1}(V) \stackrel{\delta^{D}}{\longrightarrow} \mathcal{E}_{0}(V) .
$$

The following result is a straightforward calculation. 
Proposition 3.6. The complexes (9) and (10) are special cases of the twisted de Rham detour complex (3) of Theorem 3.2.

\section{Translation via the Yang-Mills Detour Complex}

We may use the Theorem 3.2 to construct more exotic differential complexes. The ideas here are partly inspired by Eastwood's curved translation principle [21, 20] which in turn is a geometric adaptation of the Jantzen-Zuckermann translation functor from representation theory.

Consider the following general situation. Suppose that there are vector bundles (or rather section spaces thereof) $\mathcal{B}^{0}, \mathcal{B}^{1}, \mathcal{B}_{1}$ and $\mathcal{B}_{0}$ and differential operators $L_{0}$, $L_{1}, L^{1}, L^{0}, \mathcal{D}$ and $\overline{\mathcal{D}}$ which act as indicated in the following diagram:

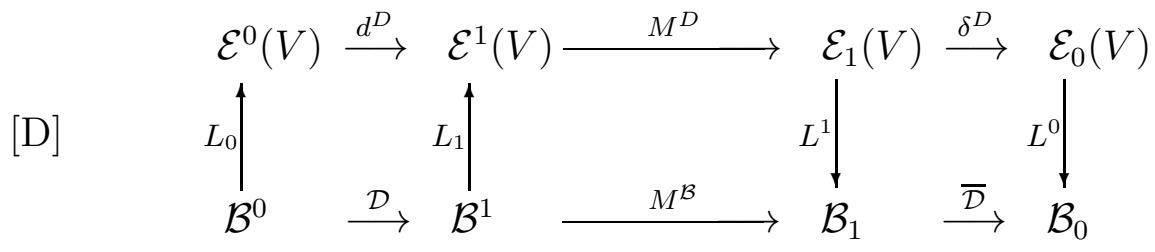

The top sequence is (3) for a connection $D$ with curvature $F$ and the operator $M^{\mathcal{B}}: \mathcal{B}^{1} \rightarrow \mathcal{B}_{1}$ is defined to be the composition $L^{1} M^{D} L_{1}$. Suppose that the squares at each end commute, in the sense that as operators $\mathcal{B}^{0} \rightarrow \mathcal{E}^{1}(V)$ we have $d^{D} L_{0}=L_{1} \mathcal{D}$ and as operators $\mathcal{E}_{1}(V) \rightarrow \mathcal{B}_{0}$ we have $L^{0} \delta^{D}=\overline{\mathcal{D}} L^{1}$. Then on $\mathcal{B}^{0}$ we have

$$
M^{\mathcal{B}} \mathcal{D}=L^{1} M^{D} L_{1} \mathcal{D}=L^{1} M^{D} d^{D} L_{0}=L^{1} \varepsilon\left(\delta^{D} F\right) L_{0},
$$

and similarly $\overline{\mathcal{D}} M^{\mathcal{B}}=-L^{0} \iota\left(\delta^{D} F\right) L_{0}$. Thus if $D$ is Yang-Mills then the lower sequence, viz.

$$
\mathcal{B}^{0} \stackrel{\mathcal{D}}{\longrightarrow} \mathcal{B}^{1} \stackrel{M^{\mathcal{B}}}{\longrightarrow} \mathcal{B}_{1} \stackrel{\overline{\mathcal{D}}}{\longrightarrow} \mathcal{B}_{0}
$$

is a complex.

Remarks: Note that if the connection $D$ preserves a Hermitian or metric structure on $V$ then we need only the single commuting square $d^{D} L_{0}=L_{1} \mathcal{D}$ on $\mathcal{B}^{0}$ to obtain such a complex; by taking formal adjoints we obtain a second commuting square $\left(L^{0} \delta^{D}=\overline{\mathcal{D}} L^{1}\right): \mathcal{B}_{1} \rightarrow \mathcal{B}_{0}$ where $\mathcal{B}_{0}$ and $\mathcal{B}_{1}$ are appropriate density twistings of the bundles dual to $\mathcal{B}^{0}$ and $\mathcal{B}^{1}$ respectively.

Obviously for (11) to be a complex, it is sufficient (and necessary) for $L^{1} M^{D}\left(d^{D} L_{0}-\right.$ $\left.L_{1} \mathcal{D}\right)$ to vanish on $\mathcal{B}^{0}$ and for $\left(\overline{\mathcal{D}} L^{1}-L^{0} \delta^{D}\right) M^{D} L_{1}$ to vanish on $\mathcal{B}^{1}$.

4.1. The complex for (almost) Einstein scales. We work in the setting of conformal $n$-manifolds, $n \geq 3$. We will construct here a diagram of the form $[D]$ via the normal conformal tractor connection. The standard tractor bundle is vector bundle with a conformally invariant connection that we may view as arising as an induced structure from the Cartan bundle and connection of [17]. In fact the Cartan connection is readily recovered from the tractor connection, see [14] where such connections and related calculus are described for the class of parabolic geometries (which also includes, for example, CR geometry, quaternionic structures 
and projective geometry). For our current construction it is not the normality of the tractor connection, in the sense of $[14,17]$, that is important. Rather the key point is that it arises from a prolongation (as observed in [3]) of a certain (finite type) partial differential operator $P$ that we may take as the operator $\mathcal{D}$ for the diagram $[\mathrm{D}]$ : In terms of a metric $g$, this operator $P$ is given by

$$
P \sigma=\mathrm{TF}\left(\nabla_{a} \nabla_{b} \sigma+\mathrm{P}_{a b} \sigma\right)
$$

where $\sigma \in \mathcal{E}[1]$. Modulo the trace part, this is the differential operator which controls the conformal transformation of the Schouten tensor. In particular a metric $\sigma^{-2} \boldsymbol{g}$ is Einstein if and only if the scale $\sigma \in \mathcal{E}[1]$ is non-vanishing and satisfies $P \sigma=0$. In order to be explicit we give a construction of the tractor connection here, as it is the key to obtaining the required commutative diagram. For further details see [13].

We write $J^{k} E[1]$ for the bundle of k-jets of germs of sections of $E[1]$. Considering, at each point of the manifold, sections which vanish to first order at the given point reveals a canonical sequence,

$$
0 \rightarrow S^{2} T^{*} M \otimes E[1] \rightarrow J^{2} E[1] \rightarrow J^{1} E[1] \rightarrow 0
$$

This is the jet exact sequence at 2-jets. Via the conformal metric $\boldsymbol{g}$, on a conformal manifold the bundle of symmetric covariant 2-tensors $S^{2} T^{*} M$ decomposes directly into the trace-free part, which we will denote $E^{1,1}$, and a pure trace part isomorphic to $E[-2]$, that is $S^{2} T^{*} M[1]=E^{1,1}[1] \oplus E[-1]$. The standard tractor bundle $\mathbb{T}$ may defined as the quotient of $J^{2} E[1]$ by the image of $E^{1,1}[1]$ in $J^{2} E[1]$. By construction this is invariant, it depends only on the conformal structure. Also by construction, it is an extension of the 1-jet bundle

$$
0 \rightarrow E[-1] \rightarrow \mathbb{T} \rightarrow J^{1} E[1] \rightarrow 0
$$

Note that there is a tautological operator $\mathbb{D}: \mathcal{E}[1] \rightarrow \mathcal{E}^{0}(\mathbb{T})$ which is simply the composition of the universal 2-jet differential operator $j^{2}: \mathcal{E}[1] \rightarrow \mathcal{E}^{0}\left(J^{2} E[1]\right)$ followed by the canonical projection $\mathcal{E}^{0}\left(J^{2} E[1]\right) \rightarrow \mathcal{E}^{0}(\mathbb{T})$. By construction this is invariant.

Via a choice of metric $g$, and the Levi-Civita connection it determines, we obtain a differential operator $\mathcal{E}[1] \rightarrow \mathcal{E}[1] \oplus \mathcal{E}^{1}[1] \oplus \mathcal{E}[-1]$ by $\sigma \mapsto\left(\sigma, \nabla_{a} \sigma,-\frac{1}{n}(\Delta+\mathrm{J}) \sigma\right)$ and this obviously determines an isomorphism

$$
\mathcal{E}^{0}(\mathbb{T}) \stackrel{g}{\cong} \mathcal{E}[1] \oplus \mathcal{E}^{1}[1] \oplus \mathcal{E}[-1]
$$

Changing to a conformally related metric $\widehat{g}=e^{2 \omega} g$ ( $\omega$ a smooth function) gives a different isomorphism, which is related to the previous by the transformation formula

$$
\left(\widehat{\sigma, \mu_{b}, \tau}\right)=\left(\sigma, \mu_{b}-\sigma \Upsilon_{b}, \tau+\boldsymbol{g}^{b c} \Upsilon_{b} \mu_{c}-\frac{1}{2} \sigma \boldsymbol{g}^{b c} \Upsilon_{b} \Upsilon_{c}\right)
$$


where $\Upsilon:=d \omega$. Now we define a connection on $\mathcal{E}[1] \oplus \mathcal{E}^{1}[1] \oplus \mathcal{E}[-1]$ by the formula

$$
\nabla_{a}\left(\begin{array}{c}
\sigma \\
\mu_{b} \\
\rho
\end{array}\right):=\left(\begin{array}{c}
\nabla_{a} \sigma-\mu_{a} \\
\nabla_{a} \mu_{b}+g_{a b} \rho+\mathrm{P}_{a b} \sigma \\
\nabla_{a} \rho-\mathrm{P}_{a b} \mu^{b}
\end{array}\right)
$$

where, on the right-hand-side $\nabla$ is the Levi-Civita connection for $g$. Obviously this determines a connection on $\mathbb{T}$ via the isomorphism (13). What is more surprising is that if we repeat this using the metric $\widehat{g}$, conformally related to $g$, in (13) and (15) we obtain the same connection on $\mathbb{T}$. This may easily be verified by, for example, directly calculating that under such a conformal change the right-hand side of (15) transforms in exactly the same way as a (1-form valued) invariant section of $\mathbb{T}$. That is it transforms according to (14). The canonical connection on $\mathbb{T}$, so constructed, depends only on the conformal structure and is known as the (normal standard) tractor connection. In what follows we will use (13) without further explicit comment. There is also a conformally invariant tractor metric $\boldsymbol{h}$ on $\mathbb{T}$ given (as a quadratic form) by $(\sigma, \mu, \rho) \mapsto \boldsymbol{g}^{-1}(\mu, \mu)+2 \sigma \rho$. This is preserved by the connection and has signature $(p+1, q+1)$ (corresponding to $\boldsymbol{g}$ of signature $(p, q))$.

Note that, given a metric $g$, through (13) the tautological invariant operator $\mathbb{D}$ from above is given by the explicit formula

$$
\mathcal{E}^{0}[1] \rightarrow \mathcal{E}^{0}(\mathbb{T}) \quad \sigma \mapsto\left(\sigma, \nabla_{a} \sigma,-\frac{1}{n}(\Delta \sigma+\mathrm{J} \sigma)\right)
$$

This is a called a differential splitting operator since through the jet projections there is conformally invariant surjection $X: \mathcal{E}(\mathbb{T}) \rightarrow \mathcal{E}[1]$ which inverts $\mathbb{D}$. There is also a differential splitting operator

$$
E: \mathcal{E}^{1,1}[1] \rightarrow \mathcal{E}^{1}(\mathbb{T}) \quad \psi_{a b} \mapsto\left(0, \psi_{a b},-(n-1)^{-1} \nabla^{b} \psi_{a b}\right)
$$

(cf. [20]). An easy calculation verifies that this also is conformally invariant. We have the following.

Proposition 4.1. With $\nabla$ denoting the tractor connection on $\mathcal{E}^{0}(\mathbb{T})$ we have

$$
\nabla \mathbb{D}=E P \text {. }
$$

as differential operators on $\mathcal{E}[1]$. For $\sigma \in \mathcal{E}[1], \mathbb{D} \sigma$ is parallel if and only if $P \sigma=0$.

Proof: The second statement is immediate from the first. A straightforward calculation verifies that either composition applied to $\sigma \in \mathcal{E}[1]$ yields

$$
\left(\begin{array}{c}
0 \\
\operatorname{TF}\left(\nabla_{a} \nabla_{b} \sigma+\mathrm{P}_{a b} \sigma\right) \\
-\frac{1}{n} \nabla_{a}(\Delta \sigma+J \sigma)-P_{a}^{c} \nabla_{c} \sigma
\end{array}\right)
$$

In fact if a section $I \in \mathcal{E}^{0}(\mathbb{T})$ is parallel then $I=\mathbb{D} \sigma$ for some $\sigma \in \mathcal{E}[1]$ so a conformal manifold with a parallel tractor is almost Einstein in the sense that it 
has a section of $\mathcal{E}[1]$ that gives an Einstein scale on an open dense subset (see [28] for further details).

Since the tractor connection is orthogonal (for the conformally invariant tractor metric $h$ given above) the formal adjoints of the operators above give another commutative square of operators. That is with

$$
\begin{aligned}
P^{*}: \mathcal{E}_{1,1}[-1] \rightarrow \mathcal{E}_{0}[-1] & \varphi_{a b} \mapsto \nabla^{a} \nabla^{b} \varphi_{a b}+\mathrm{P}^{a b} \varphi_{a b} \\
E^{*}: \mathcal{E}_{1}(\mathbb{T}) \rightarrow \mathcal{E}_{1,1}[-1] & \left(\alpha_{a}, \nu_{a b}, \tau_{a}\right) \mapsto \nu_{(a b)_{0}}+\frac{1}{n-1} \nabla_{(a} \alpha_{b)_{0}} \\
\mathbb{D}^{*}: \mathcal{E}_{0}(\mathbb{T}) \rightarrow \mathcal{E}_{0}[-1] & \left(\sigma, \mu_{b}, \rho\right) \mapsto \rho-\nabla^{a} \mu_{a}-\frac{1}{n}(\Delta \sigma+J \sigma) \\
\delta^{\nabla}: \mathcal{E}_{1}(\mathbb{T}) \rightarrow \mathcal{E}_{0}(\mathbb{T}) & \Phi_{a B} \mapsto-\nabla^{a} \Phi_{a B},
\end{aligned}
$$

where $\mathcal{E}_{1,1}$ denotes the space of sections of $E^{1,1} \otimes E[4-n]$, we have $\mathbb{D}^{*} \delta^{\nabla}=P^{*} E^{*}$ on $\mathcal{E}_{1}(\mathbb{T})$.

Finally observe that the curvature of the tractor connection, as calculated directly from (15), is

$$
\Omega_{a b}{ }^{C}{ }_{D}=\left(\begin{array}{lll}
0 & 0 & 0 \\
A_{a b}^{c} & C_{a b d}^{c} & 0 \\
0 & -A_{d a b} & 0
\end{array}\right)
$$

and hence (see e.g. [31] for further details),

$$
\nabla^{a} \Omega_{a b}{ }^{C}{ }_{D}=\left(\begin{array}{lll}
0 & 0 & 0 \\
B_{b}^{c} & (n-4) A_{b}{ }^{c}{ }_{d} & 0 \\
0 & -B_{d b} & 0
\end{array}\right)
$$

where, on the left-hand side, $\nabla$ is the Levi-Civita connection coupled with the tractor connection on End $(\mathbb{T})$ induced from (15). Let us say that a pseudoRiemannian manifold is semi-harmonic if its tractor curvature is Yang-Mills, that is $\nabla^{a} \Omega_{a b}{ }^{C}{ }_{D}=0$. Note that in dimensions $n \neq 4$ this is not a conformally invariant condition and a semi-harmonic space is a Cotton space that is also Bach-flat. From our observations above, the semi-harmonic condition is conformally invariant in dimension 4 and according to the last display we have the following result.

Lemma 4.2. In dimension 4 the tractor connection (15) is a Yang-Mills connection if and only if the structure is Bach-flat.

This result is not new and equivalent observations have been known in the literature for some time $[39,4,37]$. It brings us to the following. Let us write $M^{\mathbb{T}}$ for the composition $E^{*} M^{\nabla} E$. On $h \in \mathcal{E}^{1,1}[1]$ we have

$$
\left(M^{\mathbb{T}} h\right)_{a b}=-T F S\left(\nabla^{c}\left(\nabla_{c} h_{a b}-\nabla_{a} h_{c b}\right)-\frac{1}{n-1} \nabla_{a} \nabla^{c} h_{b c}+C_{a b}^{c}{ }^{d} h_{c d}\right),
$$

and the following results. 
Theorem 4.3. The sequence

$$
\mathcal{E}^{0}[1] \stackrel{P}{\rightarrow} \mathcal{E}^{1,1}[1] \stackrel{M^{\mathbb{T}}}{\rightarrow} \mathcal{E}_{1,1}[-1] \stackrel{P^{*}}{\rightarrow} \mathcal{E}_{0}[-1]
$$

has the following properties.

(i) It is a formally self-adjoint sequence of differential operators and, for $\sigma \in \mathcal{E}^{0}[1]$

$$
\left(M^{\mathbb{T}} P \sigma\right)_{a b}=-T F S\left(B_{a b} \sigma-(n-4) A_{a b c} \nabla^{c} \sigma\right),
$$

where TFS (...) indicates the trace-free symmetric part of the tensor concerned. In particular it is a complex on semi-harmonic manifolds.

(ii) In the case of Riemannian signature the complex is elliptic.

(iii) In dimension 4, (17) is a sequence of conformally invariant operators and it is a complex if and only if the conformal structure is Bach-flat.

Proof: Setting

$$
\mathcal{D}=P, \quad L_{0}=\mathbb{D}, \quad L_{1}=E
$$

we have the situation of the translation diagram [D] above, with the right square given by formal adjoints of these operators, and the tractor bundle connection pair $(\mathbb{T}, \nabla)$ used for $(V, D)$ in the top row. That is:

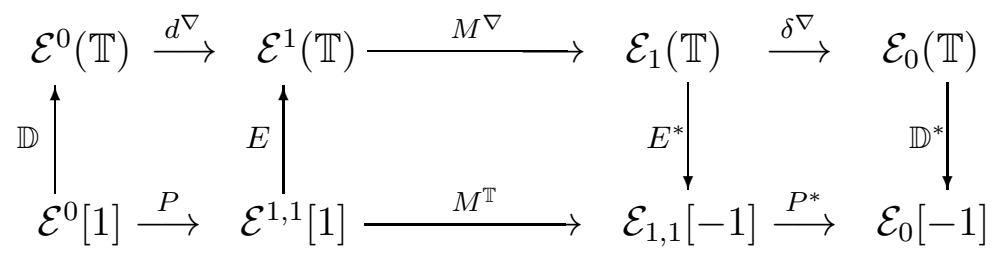

By construction the lower sequence (17) is formally self-adjoint, and in dimension 4 conformally invariant. If the structure is semi-harmonic then the upper sequence is a complex and hence, from the commutativity of the diagram, (17) is a complex. In particular on Bach-flat 4-manifolds we obtain a complex. On the other hand, from (18) it follows that in dimension 4 we obtain a complex only if the structure is Bach-flat.

From (15) we calculate $d^{\nabla}$ on the range of $E$ to obtain

$$
d^{\nabla}\left(\begin{array}{c}
0 \\
\nu \\
\tau
\end{array}\right)=\left(\begin{array}{c}
0 \\
Q \nu \\
*
\end{array}\right)
$$

where, for $\nu \in \mathcal{E}^{1,1}[1]$, we have $\tau=-\frac{1}{(n-1)} \nabla^{b} \nu_{a b}, Q$ is given by

$$
(Q \nu)_{a b c}=2 \nabla_{[a} \nu_{b] c}+2 \boldsymbol{g}_{c[a} \tau_{b]},
$$

and we do not need the details of the term indicated by $*$. It follows immediately from (19), and the formulae for the tractor metric, that we have $M^{\mathbb{T}}:=E^{*} M^{\nabla} E=$ $Q^{*} Q+\mathrm{LOT}$ where $Q^{*}$ denotes the formal adjoint of the operator $Q$. In Riemannian signature the leading symbol of $Q^{*} Q$ has the same kernel as the leading symbol of $Q$, and it follows easily that the complex is elliptic. The "lower order terms" (indicated by LOT) in $M^{\mathbb{T}}$ arise simply from the tractor curvature in the formula 
for $M^{D}$ and amount to an action by the Weyl curvature. Including this yields the explicit formula for $M^{\mathbb{T}}$ given above the Theorem. The expression (18) for the composition $M^{\mathbb{T}} P$ follows from this by a short direct calculation. (The calculation is even simpler if the result of Lemma 3.1 is imported).

Remark: Note that the formula for $M^{\mathbb{T}}$ is closely related to, but not the same as, the operator which arises from deformations of Einstein structures (for the latter see e.g. [6] and references therein). It should be valuable to expose the geometric meaning of the first cohomology of the sequence (17).

Corollary 4.4. Einstein 4-manifolds are Bach-flat.

Proof: If a non-vanishing density $\sigma$ is an Einstein scale then, calculating in that scale, we have $M^{\mathbb{T}} P \sigma=-B \sigma$, where $B$ is the Bach tensor. On the other hand if $\sigma$ is an Einstein scale then $P \sigma=0$ (see (12)).

Remarks: In fact, more generally, almost Einstein manifolds are also necessarily Bach flat. Since an almost Einstein manifold has an Einstein scale on an open dense subspace, this follows by continuity of the Bach tensor. (The higher dimensional extension of this result is that even dimensional almost Einstein manifolds have vanishing Fefferman-Graham obstruction tensor, see e.g. [28] and references therein.) The result that Einstein metrics are Bach-flat is well-known by other means (see e.g. [38, 31]). Nevertheless we feel the detour complex gives an interesting route to this. In any dimension Einstein metrics satisfy $P_{a b}=\frac{1}{n} J g_{a b}$ with $J$ constant, so it follows from the definitions of the Cotton tensor and the Bach tensor (2) that Einstein metrics are semi-harmonic. Thus there are many examples of semi-harmonic manifolds.

4.2. The twistor spinor complex. We assume here that we have a conformal spin structure. This is no restriction locally. For the purpose of being selfcontained and having the results in a uniform notation we derive the basic spinor identities we require. An alternative treatment for many of these may be found in, e.g. [26]. We will use the spin-tractor connection below. This is often termed the local twistor connection $[41,5]$. The notation we use (and the basic tractor tools) follows [9] which presents a spin-tractor calculus developed by the first author and Branson. Following that source we write $\mathbb{S}$ for the basic spinor bundle and $\overline{\mathbb{S}}=\mathbb{S}[-n]$ (i.e. the bundle that pairs globally in an invariant way with $\mathbb{S}$ on conformal $n$-manifolds). Evidently the weight conventions here give $\mathbb{S}$ a "neutral weight". In terms of, for example, the Penrose weight conventions $\mathbb{S}=E^{\lambda}\left[-\frac{1}{2}\right]=E_{\lambda}\left[\frac{1}{2}\right]$, where $E^{\lambda}$ denotes the basic contravariant spinor bundle in [41].

We write $\mathrm{Tw}$ for the so-called twistor bundle, that is the subbundle of $T^{*} M \otimes$ $\mathbb{S}[1 / 2]$ consisting of form spinors $u_{a}$ such that $\gamma^{a} u_{a}=0$, where $\gamma_{a}$ is the usual Clifford symbol. We use $\mathbb{S}$ and Tw also for the section spaces of these bundles. The twistor operator is the conformally invariant Stein-Weiss gradient

$$
\mathbf{T}: \mathbb{S}[1 / 2] \rightarrow \mathrm{Tw}
$$

given explicitly by

$$
\psi \mapsto \nabla_{a} \psi+\frac{1}{n} \gamma_{a} \gamma^{b} \nabla_{b} \psi
$$


The main result of this section is that this completes to a differential complex as follows.

Theorem 4.5. On semi-harmonic pseudo-Riemannian $n$-manifolds $n \geq 4$ we have a differential complex

$$
\mathbb{S}[1 / 2] \stackrel{\mathbf{T}}{\rightarrow} \mathrm{Tw} \stackrel{M^{\Sigma}}{\rightarrow} \overline{\mathrm{Tw}} \stackrel{\mathbf{T}^{*}}{\rightarrow} \overline{\mathbb{S}}[-1 / 2]
$$

where $\mathbf{T}$ is the usual twistor operator, $\mathbf{T}^{*}$ its formal adjoint, and $M^{\Sigma}$ is a third order operator and given by the formula (26) below. The sequence is formally self-adjoint and in the case of Riemannian signature the complex is elliptic.

In dimension 4 the sequence (20) is conformally invariant and it is a complex if and only if the conformal structure is Bach-flat.

Remarks: Of course on a fixed pseudo-Riemannian manifold we may ignore the conformal weights.

Note also that, for example in dimension 4, under the chirality decomposition of this sequence, we get the two complexes

$$
\mathbb{S}_{ \pm}[1 / 2] \stackrel{\mathbf{T}}{\rightarrow} \mathrm{Tw}_{ \pm} \stackrel{M^{\Sigma}}{\rightarrow} \overline{\mathrm{Tw}}_{\mp} \stackrel{\mathbf{T}^{*}}{\rightarrow} \overline{\mathbb{S}}_{\mp}[-1 / 2]
$$

by the restriction of the operators $\mathbf{T}, \mathbf{T}^{*}$, and $M^{\Sigma}$.

If we were to apply the construction below in dimension 3 then we would obtain a trivial operator $M^{\Sigma}$. In this dimension the BGG sequence (see section 2) takes the form (20), where the middle operator is of second order.

In the calculations which follow it will often be convenient to use abstract indices for the form bundles while at the same time not using any indices for the spinor bundles. We have already done this implicitly above, for example in the formula for the twistor operator which, in this notation, acts $\mathbf{T}_{a}: \mathbb{S}[1 / 2] \rightarrow \mathrm{Tw}_{a}$. From the usual gamma matrices $\gamma^{a}$ satisfying

$$
\gamma^{a} \gamma^{b}+\gamma^{b} \gamma^{a}=-2 \boldsymbol{g}^{a b} \mathrm{Id}
$$

we switch to the symbols $\beta:=\gamma / \sqrt{2}$, so that

$$
\beta^{a} \beta^{b}+\beta^{b} \beta^{a}=-\boldsymbol{g}^{a b} \mathrm{Id},
$$

this simplifies certain formulae in the following discussion. We denote the corresponding Dirac operator by $\boldsymbol{D}:=\beta^{a} \nabla_{a}$.

Given a metric $g$ from the conformal class the spin-tractor bundle $\Sigma$ is given by

$$
\Sigma \stackrel{g}{\cong} \mathbb{S}[1 / 2] \oplus \mathbb{S}[-1 / 2]
$$

where $\mathbb{S}$. In the conformally related metric $\widehat{g}=e^{2 \omega} g$ we have a similar isomorphism and

$$
\left(\begin{array}{c}
\widehat{\psi} \\
\widehat{\varphi}
\end{array}\right)=\left(\begin{array}{c}
\psi \\
\varphi+\Upsilon_{c} \beta^{c} \psi
\end{array}\right)
$$


where $\Upsilon=d \omega$. In terms of the $g$-splitting the normal conformal spin-tractor connection is given by

$$
\nabla_{a}\left(\begin{array}{c}
\psi \\
\varphi
\end{array}\right)=\left(\begin{array}{c}
\nabla_{a} \psi+\beta_{a} \varphi \\
\nabla_{a} \varphi+P_{a b} \beta^{b} \psi
\end{array}\right) .
$$

On the right side $\nabla$ means the usual Levi-Civita (spin) connection, while on the left the same notation is used for the spin-tractor connection. It is an easy exercise to verify directly that this is a conformally invariant connection. The normality follows from the characterisation of normal tractor connections (for irreducible parabolic geometries) given in Theorem 1.3 of [13].

The invariant pseudo-Hermitian form on spin-tractors is given by

$$
\langle\varphi, \underline{\psi}\rangle+\langle\psi, \underline{\varphi}\rangle
$$

for a pair spin-tractors $(\varphi, \psi),(\underline{\varphi}, \underline{\psi})$, and where $\langle\cdot, \cdot\rangle$ is the usual Hermitian form on spinors (which is compatible with Clifford multiplication and is preserved by the Levi-Civita spin connection). It is readily verified that this is invariant under the transformations (22) and that it is preserved by the spin-tractor connection (23). We subsequently calculate in a metric scale $g$ without further comment.

We construct two differential splitting operators:

$$
L_{0}: \mathbb{S}[1 / 2] \rightarrow \mathcal{E}^{0}(\Sigma)
$$

is given by

$$
\begin{aligned}
& \psi \mapsto\left(\begin{array}{c}
\psi \\
\frac{2}{n} \boldsymbol{D} \psi
\end{array}\right) ; \\
& L_{1}: \mathrm{Tw} \rightarrow \mathcal{E}^{1}(\Sigma)
\end{aligned}
$$

is given by

$$
\psi_{a} \mapsto\left(\begin{array}{c}
\psi_{a} \\
\frac{2}{n-2}\left(\boldsymbol{D} \psi_{a}-\frac{1}{n-1} \beta_{a} \nabla^{b} \psi_{b}\right)
\end{array}\right) .
$$

It is a straightforward exercise to verify that these transform according to (22) and so are expressions, in the metric scale $g$, for conformally invariant operators. An essential feature of these operators is the following commutativity result.

Proposition 4.6. With $\nabla$ denoting the spin-tractor connection on $\mathcal{E}^{0}(\Sigma)$ we have

$$
d^{\nabla} L_{0}=L_{1} \mathbf{T}
$$

as differential operators on $\mathbb{S}[1 / 2]$. For $\psi \in \mathbb{S}[1 / 2], L_{0} \psi$ is parallel if and only if $\mathbf{T} \psi=0$.

The last comment follows immediately from the commutativity of the square given that $L_{1}$ is a differential splitting operator (and so, in particular, $L_{1} \psi_{a}=0 \Rightarrow \psi_{a}=$ $0)$. A correspondence between parallel spin-tractors and twistor spinors dates back to [25]. The extra information in the proposition is that the operator $L_{1}$ is 
a conformally invariant tractor splitting operator. We shall postpone the proof of Proposition 4.6, as we prefer to first complete the proof of Theorem 4.5.

Suppose that for a metric $g$, from the conformal class, the tractor connection is semi-harmonic. Recall this is exactly the condition that the normal tractor connection is Yang-Mills. It follows immediately that the spin-tractor connection is also Yang-Mills, since this is induced from the same principal connection simply pulled back to the 2-1 covering $\operatorname{Spin}(p+1, q+1)$-principal bundle. (Equivalently they arise from the same Cartan connection, this is the usual picture [14] and sufficient to see this result. From the Cartan picture one may easily extend to a principal bundle and connection from which the tractors are induced, and this is simply an alternative framework.) As observed above, in dimension 4 the YangMills condition is exactly the condition that the metric (or conformal structure) is Bach-flat. Thus, from the Proposition, the first part of Theorem 4.5 follows immediately from the commutative diagram below where

$$
M^{\Sigma}=L^{1} M^{\nabla} L_{1}
$$

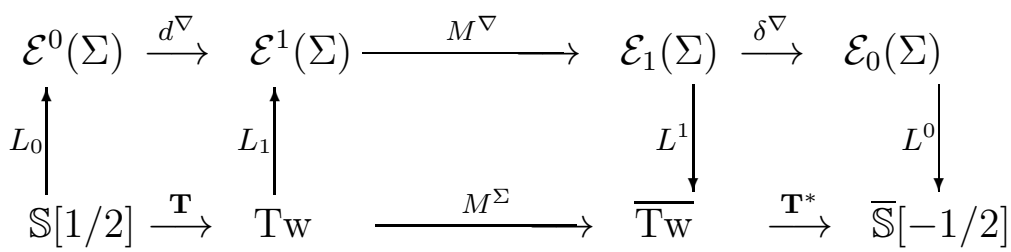

Using that $\Sigma$ is a self-dual bundle, the operators in the square at the right end of the diagram are defined as the formal adjoints of the operators in the first square. So all squares commute and both horizontal sequences are formally self-adjoint.

To establish ellipticity we require the leading term of the operator $M^{\Sigma}$. Applying the spin-tractor twisted exterior derivative to $L_{1} t$, for $t \in \mathrm{Tw}$, we obtain a result of the form

$$
\left(\begin{array}{c}
\mathbf{K} t \\
\ell \mathbf{D} \mathbf{K} t+m \beta \delta^{\nabla} \mathbf{K} t+\text { curvature }
\end{array}\right)
$$

with $\mathbf{K} t$ given by

$$
2 \nabla_{a_{1}} t_{a_{2}}+\frac{4}{n-2} \beta_{a_{1}}\left(\boldsymbol{D} t_{a_{2}}-\frac{1}{n-1} \beta_{a_{2}} \nabla^{c} t_{c}\right),
$$

where the indices $a_{1} a_{2}$ are implicitly skewed over, $\ell$ and $m$ are constants, and $\delta^{\nabla}$ is the spin-Levi-Civita connection twisted interior derivative. By construction $\mathbf{K}$ is an invariant operator $\mathbf{K}: \mathrm{Tw} \rightarrow \mathrm{Tw}^{2}$, where $\mathrm{Tw}^{2}$ is the subbundle of $E^{2} \otimes$ $\mathbb{S}[1 / 2]$ consisting of spin-forms annihilated by interior multiplication by $\beta$. It is a straightforward exercise (or one may use the BGG machinery of [15]) to construct a differential splitting operator $L_{2}: \mathrm{Tw}^{2} \rightarrow E^{2} \otimes \Sigma$. This has the form $s \mapsto$ $\left(s, \ell \boldsymbol{D} s+m \beta \delta^{\nabla} s\right.$ ) (cf. $L_{1}$ ) where $\ell \neq 0$. On the other hand in the conformally flat case it follows easily, from the uniqueness of conformal differential operators, that $L_{2} \mathbf{K}=d^{\nabla} L_{1}$. Thus we obtain the form of the bottom slot of (27). 
Since the leading term of $M^{\Sigma}$ is obtained by composing $d^{\nabla} L_{1}$ with its formal adjoint, and $\boldsymbol{D}$ is formally self-adjoint, it follows that the leading term of the operator $M^{\Sigma}$ is of the form $\mathbf{K}^{*} \pi_{2} \mathbf{D K}$. Here $\mathbf{K}^{*}$ denotes the formal adjoint of $\mathbf{K}$ and the projection $\pi_{2}$ is the projection of spinor-valued two-forms to the space $T w^{2}$.

The operator $R_{2}=\pi_{2} D$ on $T w^{2}$ is an elliptic and self adjoint operator, it is a higher spin analogue of the Dirac operator. Similarly, if $\pi_{1}$ denotes the projection of spinor-valued one-forms to $T w$, the operator $\mathbb{R}_{1}=\pi_{1} D$ is an elliptic self adjoint operator on $T w$ (usually called the Rarita-Schwinger operator). Moreover, $R_{1} \mathbf{K}^{*}$ is a multiple of $\mathbf{K}^{*} R_{2}$. Hence the leading term of the operator $M^{\Sigma}$ is a multiple of $R_{1} \mathbf{K}^{*} \mathbf{K}$.

Since elements of $\mathrm{Tw}^{2}$ are annihilated by interior Clifford multiplication, it follows from the formula for $\mathbf{K}$ that the symbol $\sigma_{\xi}\left(\mathbf{K}^{*}\right)$ is simply interior multiplication by $\xi, \iota(\xi)$.

Without loss of generality we may suppose that $\xi$ is a unit vector. It is well known that the Rarita-Schwinger operator (on the flat space) can be composed with a third order constant coefficient operator to give the square of the Laplace operator. Hence the symbol of $\mathbb{R}_{1}$ can be multiplied on the left to get $|\xi|^{4}=1$. By this left multiplication, $\sigma_{\xi}\left(\mathbf{K}^{*} R_{2} \mathbf{K}\right) t=\sigma_{\xi}\left(R_{1} \mathbf{K}^{*} \mathbf{K}\right) t=0$ implies $\iota(\xi) \sigma_{\xi}(\mathbf{K}) t=0$. Now the explicit formula for $\sigma_{\xi}(\mathbf{K}) t$ is

$$
\varepsilon(\xi) t+\frac{2}{n-2} \varepsilon(\beta)\left(\beta_{\xi} t-\frac{1}{n-1} \varepsilon(\beta) \iota(\xi) t\right)
$$

where $\varepsilon(\cdot)$ indicates exterior multiplication.

Contracting with $\xi$ and setting to zero we obtain $((n-3)$ times $)$

$$
(n-1) t=n\left(\varepsilon(\xi) \iota(\xi) t+\frac{2}{n} \varepsilon(\beta) \beta_{\xi} \iota(\xi) t\right) .
$$

The right hand side here is a multiple of $\sigma_{\xi}(\mathbf{T}) \iota(\xi) t$. Thus $t$ is in the range of $\sigma_{\xi}(\mathbf{T})$, as required.

Completing the proof and remarks: From (16), (24), and Lemma (3.1) it follows easily that in dimension 4 the composition $M^{\Sigma} \mathbf{T}$ on $\varphi$, a section of $\mathbb{S}[1 / 2]$, is, up to a non-zero multiple, a Clifford multiplication of the Bach tensor $B_{a b} \beta^{b} \varphi$. Thus the formally self-adjoint sequence (4.5) is a complex if and only if the structure is Bach-flat, as claimed in the theorem. If $\varphi$ is a twistor spinor (i.e. $\mathbf{T} \varphi=0$ ) then this Clifford action of the Bach tensor on $\varphi$ obviously vanishes. In Riemannian signature it is in fact straightforward to recover a parallel standard tractor from the parallel spin-tractor corresponding to a twistor spinor. Thus Riemannian manifolds admitting a twistor spinor are almost Einstein and so Bach flat. In fact the last conclusion here is well-known [5].

Proof of Proposition 4.6: Let $\psi$ be a section of $\mathbb{S}[1 / 2]$. Using the formula (24) for the splitting operator and the expression (23) for the spin-tractor connection 
we have

$$
\nabla_{a} L_{0} \psi=\nabla_{a}\left(\begin{array}{c}
\psi \\
\frac{2}{n} \boldsymbol{D} \psi
\end{array}\right)=\left(\begin{array}{c}
\nabla_{a} \psi+\frac{2}{n} \beta_{a} \boldsymbol{D} \psi \\
\frac{2}{n} \nabla_{a} \boldsymbol{D} \psi+P_{a b} \beta^{b} \psi
\end{array}\right)
$$

Recalling that $\gamma=\sqrt{2} \beta$ and that $\boldsymbol{D}=\beta^{a} \nabla_{a}$, we thus have

$$
\nabla_{a} L_{0} \psi=\left(\begin{array}{c}
\mathbf{T}_{a} \psi \\
\frac{2}{n} \nabla_{a} \boldsymbol{D} \psi+P_{a b} \beta^{b} \psi
\end{array}\right) .
$$

From (25) it is clear that it remains to show that

$$
\frac{2}{n} \nabla_{a} \boldsymbol{D} \psi+P_{a b} \beta^{b} \psi=\frac{2}{n-2}\left(\boldsymbol{D} \mathbf{T}_{a} \psi-\frac{1}{n-1} \beta_{a} \nabla^{b} \mathbf{T}_{b} \psi\right) .
$$

Let us note some simpler identities first. First for the Levi-Civita spin-connection the curvature on a spinor $\varphi$ is given by

$$
\left[\nabla_{a}, \nabla_{b}\right] \varphi=-\frac{1}{2} R_{a b c d} \beta^{c} \beta^{d} \varphi,
$$

where $R$ is the usual Riemannian curvature. Then from the Bianchi identities and the Clifford relation (21) we get

$$
R_{a b c d} \beta^{b} \beta^{c} \beta^{d}=\operatorname{Ric}_{a b} \beta^{b} \quad R_{a b c d} \beta^{a} \beta^{b} \beta^{c} \beta^{d}=-\mathrm{Sc} / 2
$$

Next an elementary calculation shows that $\mathbf{T}^{*}$, the formal adjoint of $\mathbf{T}$, is given on $T w$ by $\varphi_{a} \mapsto-\nabla^{a} \varphi_{a}$. Thus, for $\psi$ in $\mathbb{S}[1 / 2]$,

$$
-\mathbf{T}^{*} \mathbf{T} \psi=\Delta \psi+\frac{2}{n} \boldsymbol{D}^{2} \psi
$$

(where $\Delta:=\nabla^{a} \nabla_{a}$ ) since of the spin-connection preserves the Clifford symbols. On the other hand since $\boldsymbol{D}^{2}=\beta^{a} \nabla_{a} \beta^{b} \nabla_{b}=\beta^{a} \beta^{b} \nabla_{a} \nabla_{b}$ and the $\beta$ s anti-commute up to a trace, as in (21), while the $\nabla$ s commute up to curvature we obtain

$$
\boldsymbol{D}^{2} \psi=-\frac{1}{2} \Delta \psi-\frac{1}{4} R_{a b c d} \beta^{a} \beta^{b} \beta^{c} \beta^{d} \psi
$$

and so using (29) we come to

$$
\Delta \psi=-2 \boldsymbol{D}^{2} \psi+\frac{1}{4} \mathrm{Sc} \cdot \psi
$$

This with the expression above for $\mathbf{T}^{*} \mathbf{T}$ gives

$$
-\mathbf{T}^{*} \mathbf{T} \psi=2\left(\frac{1-n}{n}\right) \boldsymbol{D}^{2} \psi+\frac{1}{4} \mathrm{Sc} \cdot \psi \text {. }
$$

We are now ready to calculate the left-hand side of (28). Applying $\beta^{b} \nabla_{b}$ to the defining identity $\nabla_{a} \psi=-\frac{2}{n} \beta_{a} \boldsymbol{D} \psi+\mathbf{T}_{a} \psi$ we get

$$
\beta^{b} \nabla_{b} \nabla_{a} \psi=-\frac{2}{n} \beta^{b} \beta_{a} \nabla_{b} \boldsymbol{D} \psi+\beta^{b} \nabla_{b} \mathbf{T}_{a} \psi
$$


Commuting the derivatives on the left and writing $\boldsymbol{D}$ as a shorthand for $\beta^{b} \nabla_{b}$ (applied to e.g. $\mathbf{T} \psi$ ), we obtain

$$
\nabla_{a} \boldsymbol{D} \psi+\frac{1}{2} R_{a b c d} \beta^{b} \beta^{c} \beta^{d} \psi=\frac{2}{n} \nabla_{a} \boldsymbol{D} \psi+\frac{2}{n} \beta_{a} \boldsymbol{D}^{2} \psi+\boldsymbol{D T}_{a} \psi
$$

Next rearranging the terms and using (29) gives

$$
\left(\frac{n-2}{n}\right) \nabla_{a} \boldsymbol{D} \psi=\frac{2}{n} \beta_{a} \boldsymbol{D}^{2} \psi-\frac{1}{2} \operatorname{Ric}_{a b} \beta^{b} \psi+\boldsymbol{D} \mathbf{T}_{a} \psi
$$

Using now the identity (30) from above to substitute for $\boldsymbol{D}^{2} \psi$ yields

$$
\left(\frac{n-2}{n}\right) \nabla_{a} \boldsymbol{D} \psi=-\frac{1}{2}\left(\operatorname{Ric}_{a b}-\frac{1}{2(n-1)} \operatorname{Sc} \boldsymbol{g}_{\mathrm{ab}}\right) \beta^{\mathrm{b}} \psi+\boldsymbol{D} \mathbf{T}_{\mathrm{a}} \psi+\frac{1}{\mathrm{n}-1} \beta_{\mathrm{a}} \mathbf{T}^{*} \mathbf{T} \psi \text {. }
$$

But multiplying this through with $2 /(n-2)$ and using that the Schouten tensor $P_{a b}=\frac{1}{n-2}\left(\operatorname{Ric}_{a b}-\frac{1}{2(n-1)} \operatorname{Sc} \boldsymbol{g}_{\mathrm{ab}}\right)$, and once again that $\mathbf{T}^{*} \varphi=-\nabla^{a} \varphi_{a}$, this gives exactly the expression (28), which is thus seen to be an identity.

\section{REFERENCES}

[1] M.F. Atiyah, N. Hitchin, and I.M. Singer, Self-duality in four-dimensional Riemannian geometry, Proc. Roy. Soc. London Ser A 362, (1978) 425-461.

[2] R. Bach, Zur Weylschen Relativitätstheorie und der Weylschen Erweiterung des Krümmungstensorbegriffs, Math. Z. 9, (1921) 110-135.

[3] T.N. Bailey, M.G. Eastwood, and A.R. Gover, Thomas's structure bundle for conformal, projective and related structures, Rocky Mountain J. Math. 24, (1994) 1191-1217.

[4] R.J. Baston and L.J. Mason, The conformal Einstein equations, in: "Further advances in twistor theory: Volume II: Integrable systems, conformal geometry and gravitation" L.J. Mason, L.P. Hughston and P.Z. Kobak (eds.), Longman, Essex, 1995.

[5] H. Baum, T. Friedrich, R. Grunewald, and I. Kath, Twistors and Killing spinors on Riemannian manifolds, Teubner-Texte zur Mathematik, 124. B.G. Teubner Verlagsgesellschaft mbH, Stuttgart, 1991. 180 pp.

[6] A.L. Besse, Einstein manifolds, Ergebnisse der Mathematik und ihrer Grenzgebiete (3), 10, Springer-Verlag, Berlin, 1987. xii+510pp.

[7] B.D. Boe, and D.H. Collingwood, A comparison theory for the structure of induced representations, II. Math. Z. 190, (1985) 1-11.

[8] T. Branson, Sharp inequalities, the functional determinant, and the complementary series. Trans. Amer. Math. Soc., 347, (1995) 3671-3742.

[9] T. Branson, Conformal Structure and spin geometry in: Dirac operators: yesterday and today, Proceedings of the Summer School and Workshop held in Beirut, August 27-September 7, 2001. Edited by J.-P. Bourguignon, T. Branson, A. Chamseddine, O. Hijazi and R.J. Stanton. International Press, Somerville, MA, 2005. viii+310 pp.

[10] T. Branson, Q-curvature and spectral invariants, Rend. Circ. Mat. Palermo (2) Suppl. No. 75 (2005), 11-55.

[11] T. Branson, and A.R. Gover, Conformally invariant operators, differential forms, cohomology and a generalisation of Q-curvature, Comm. Partial Differential Equations, 30, (2005) $1611-1669$.

[12] T. Branson, A.R. Gover, The conformal deformation detour complex for the obstruction tensor, Proc. Amer. Math. Soc., 135, (2007) 2961-2965.

[13] A. Cap, and A.R. Gover, Tractor bundles for irreducible parabolic geometries, in: Global analysis and harmonic analysis (Marseille-Luminy, 1999), 129-154, Sémin. Congr., 4, Soc. Math. France, Paris, 2000. 
Gover, Somberg, Souček

[14] A. Čap, and A.R. Gover, Tractor calculi for parabolic geometries, Trans. Amer. Math. Soc., 354, (2002) 1511-1548.

[15] A. Čap, J. Slovák, V. Souček, Bernstein-Gelfand-Gelfand sequences, Annals of Math., 154, (2001) 97-113.

[16] A. Cap, and V. Souček, Subcomplexes in Curved BGG-Sequences. Preprint math.DG/0508534, http://www.arxiv.org

[17] E. Cartan, Les espaces à connexion conforme, Ann. Soc. Pol. Math. 2, (1923) 171-202.

[18] S.-Y. A. Chang, Non-linear elliptic equations in conformal geometry, Zurich Lectures in Advanced Mathematics, European Mathematical Society, Zürich, 2004, viii+92 pp

[19] S.K. Donaldson, Floer homology groups in Yang-Mills theory, Cambridge Tracts in Mathematics, 147, Cambridge University Press, Cambridge, 2002. viii+236 pp.

[20] M. Eastwood, Notes on conformal differential geometry, Rend. Circ. Mat. Palermo (2) Suppl. No. 43 (1996), 57-76.

[21] M.G. Eastwood and J.W. Rice, Conformally invariant differential operators on Minkowski space and their curved analogues, Comm. Math. Phys. 109, (1987) 207-228; Erratum: Comm. Math. Phys. 144, (1992) 213.

[22] M.G. Eastwood, J. Slovák, Semiholonomic Verma modules, J. Alg., 197, (1997) 424-448.

[23] C. Fefferman, C.R. Graham, Conformal invariants, in Elie Cartan et les mathématiques d'aujourd'hui, Astérisque, hors série (SMF, Paris, 1985) 95-116.

[24] C. Fefferman, C.R. Graham, Q-curvature and Poincaré metrics, Math. Res. Lett., 9, (2002) $139-151$.

[25] T. Friedrich, On the conformal relation between twistors and Killing spinors, Rend. Circ. Mat. Palermo (2) Suppl. No. 22, (1989), 59-75.

[26] T. Friedrich, Dirac-Operatoren in der Riemannschen Geometrie, Mit einem Ausblick auf die Seiberg-Witten-Theorie, Advanced Lectures in Mathematics. Friedr. Vieweg \& Sohn, Braunschweig, 1997. xii+207pp.

[27] A.R. Gover, Aspects of parabolic invariant theory, Rend. Circ. Mat. Palermo (2) Suppl. No. $59,(1999)$ 25-47.

[28] A.R. Gover, Almost conformally Einstein manifolds and obstructions. Differential geometry and its applications, 247-260, Matfyzpress, Prague, 2005. Electronic: math.DG/0412393, http://www.arxiv.org

[29] A.R. Gover, Laplacian operators and Q-curvature on conformally Einstein manifolds, Math. Ann., 336, 311-334.

[30] A.R. Gover, and F. Leitner, A sub-product construction of Poincare-Einstein metrics, preprint math.DG/0608044, http://www.arxiv.org.

[31] A.R. Gover and P. Nurowski, Obstructions to conformally Einstein metrics in $n$ dimensions, J. Geom. Phys., 56, (2006) 450-484.

[32] A.R. Gover and L.J. Peterson, Conformally invariant powers of the Laplacian, Q-curvature, and tractor calculus, Commun. Math. Phys. 235 (2003) 339-378.

[33] A.R. Gover and J. Šilhan, The conformal Killing equation on forms - prolongations and applications, Diff. Geom. Applic., to appear. Preprint math.DG/0601751, http://arXiv.org.

[34] C.R. Graham and K. Hirachi, The ambient obstruction tensor and Q-curvature, in AdS/CFT correspondence: Einstein metrics and their conformal boundaries, 59-71, IRMA Lect. Math. Theor. Phys., 8, Eur. Math. Soc., Zürich, 2005.

[35] C.R. Graham, R. Jenne, L. Mason, G. Sparling, Conformally invariant powers of the Laplacian, I: existence, J. London Math. Soc., 46, (1992) 557-565.

[36] C.R. Graham, and M. Zworski, Scattering matrix in conformal geometry, Invent. Math., 152, (2003) 89-118.

[37] M. Korzynski, J. Lewandowski, The normal Cartan connection and the Bach tensor, Class. Quantum Gravity, 20, (2003) 3745-3764.

[38] C. Kozameh, E.T. Newman, K.P. Tod Conformal Einstein Spaces GRG 17, (1985) 343-352. 
[39] S. Merkulov, A conformally invariant theory of gravitation and electromagnetism, Class. Quantum Gravity, 1, (1984), 349-354.

[40] J.M. Lee, T.H. Parker, The Yamabe problem, Bull. Amer. Math. Soc., 17, (1987) 37-91.

[41] R. Penrose, and W. Rindler, Wolfgang, Spinors and space-time, Vol 1 and Vol. 2. Spinor and twistor methods in space-time geometry, Cambridge Monographs on Mathematical Physics Cambridge University Press, Cambridge, 1987, 1988, x+458pp, x+501 pp.

ARG: Department of Mathematics, The University of Auckland, Private Bag 92019, Auckland 1, New Zealand

E-mail address: gover@math.auckland.ac.nz

PS and VS: Mathematical Institute, Faculty of Mathematics and Physics, Charles University, Sokolovská 83, 18675 Praha, Czech Republic

E-mail address: somberg@karlin.mff.cuni.cz, soucek@karlin.mff.cuni.cz 